\title{
Generalising the Entropy Formula through Master Equations
}

Entropy is one of the most
important and most widely
studied quantities in physics,
and for centuries, its value
has been robustly described
using simple mathematical
relationships. Yet however
elegant, Tamás Biró at the
Hungarian Academy of
Science believer the formula
is hiding a more complex
array of relationships. Through
constructing 'master equations'
to describe these relationships,
Biró and his colleagues are using
statistics to study problems
as diverse as the formation of
hadrons, changes in biodiversity,
and patterns in popularity
on Facebook.

- a different degree of chaos associated with it. While atom within metals are arranged in neat, highy orded latices, the novements of gas molecules in the air are far more $19^{\text {th }}$ century physicists have described this chaos using a quantity known as 'entropy'. The changes in entropy which 'take place during physical processes fo the basis of one of the most fundamenta laws of physics: The Second Law of Thermodynamics. The law states that in any system closed off from the outside, entropy must not decrease over time; as such, the universe becomes more chao as a whole.

As Dr Biró describes, this inevitable increase in entropy represents a decrease in the number of possible leftover arrangements of particles, or 'states', that a system can find itself in as the "Macroscopically, entropy has proved never to decrease spontaneously; an idea related to the 'arrow of time', or irreversibility," he explains. "Many processes in nature end up only

Energy, Entropy, the $2^{\text {nd }}$ law of Thermodynamics

000

$00_{0}^{0} 0_{0}^{\circ} 0_{0}^{0} 0_{0}^{0}$

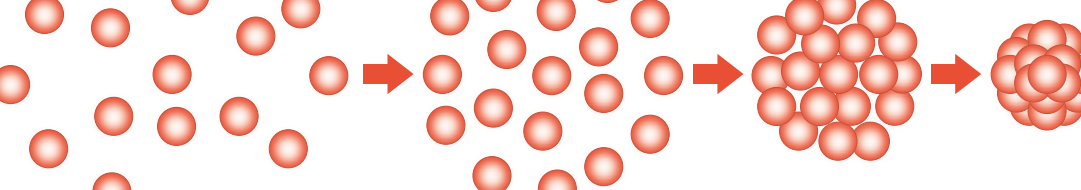
O 0

Low Randomness,
Low Entropy, Low Disorder
High Randomness,
High Entropy, High Disorder in some selected states while they may begin in any of a vastly larger amount of possible states. All microscopic changes in a complicated closed system conspire in a way that the total entropy

To quantify entropy, physicists ultimately need to use statistics to study how

Prangements over time "Kinetic theoy

and its follower, statistical physics, depend on our knowledge about entropy," Dr Biró continues. "They can be viewed as given mathematical formulae based on the probability of being in a given state. All random, or 'stochastic' models are now checked for satisfying such a theorem, accounting for random forces in their calculations." Even after centures of scrutiny, these mathematica descriptions have proved time and again to robustly describe real physical Biró and his colleagues rewearch, Dr the theory a step further.

\section{GENERALISIN}

TZMANN'S FORMULA

In the 1870s, Austrian physicist Ludwig quantity of entropy down to a remarkably simple, elegant formula. He proposed that its value simply has a logarithmic relationship with the number of possible states a system can have under its current conditions. Yet although the formula is so effective in mathematically predicting changes in entropy, Dr Biro and colleagues believe it cannot completely account for the more complex it In their recesses which underlay t. In their research, he researchers aim to generalize Bolrmann's simple

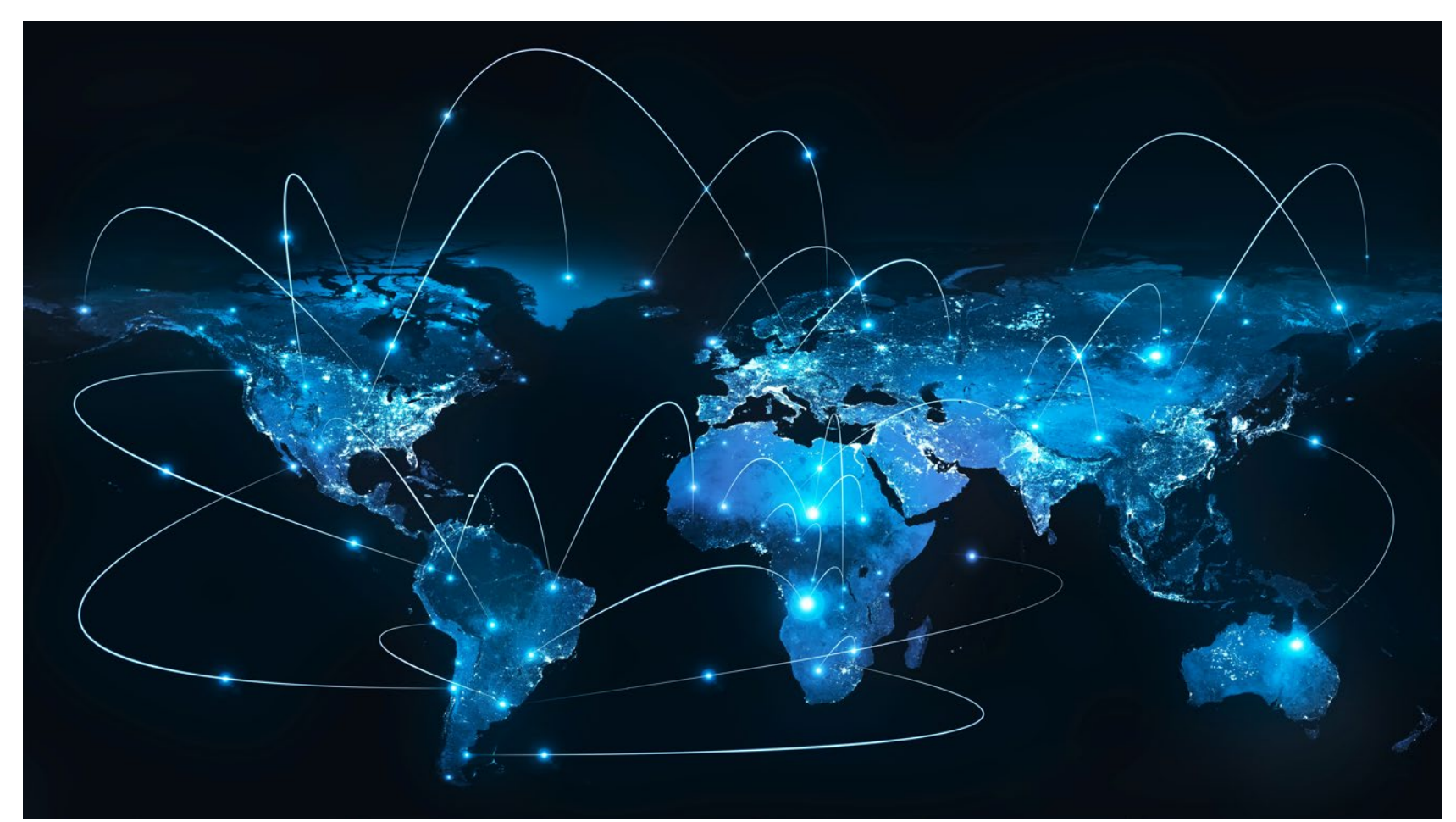

relationships which build up to the overall logarithmic relationship.

"Our research couples to the novel efforts to generalize Boltzmann's original formula containing the logarithm - a neat function mapping products to sums," says Dr Biró. "What are the more general dynamical processes which make it unavoidable to use Boltzmann's logarithm?" Through several studies, the researchers have used real-world examples of entropy changes to study how Boltzmann's logarithm can be generalised in this way. The problens fhey have analysed so far range from cattenal a

\section{SOLVING THE}

HADRONIZATION PROBLEM

In the dynamic first moments of the

universe, fundamental particles known as quarks and gluons existed as freelyflowing elements of a gas-like soup or plasma. However, these particles ultimately cannot exist by themselves; within octillionths of a second after the Big Bang, they grouped together to form stable structures named hadrons - made from three quarks bound together by gluons. Yet the process of hadronization' throws up a problem for physicists: since hadrons appear to be far more ordered than a quark-gluon plasma, how could the overall entropy of
have increased?
All microscopic changes in a complicated closed system conspire in a way that the total entropy never decreases. In their research, Dr Biro and colleagues have proposed a new way of looking at the problem, based on the earlier work of Brazilian physicist Constantino Tsallis. In the 1980s, Tsallis drew up a new set of statistical parameters to generalise Boltzmann's formula, resulting in a case of entropy. In particular, his equations case of entropy. In particular, his equation distributions statistical formula describe how likely it is for individal

Number Dynamics place. The probability distributions derived by Tsallis could describe the entropy of physical systems far more

Based on Tsallis' work, Dr Birós team could construct a set of 'master equations'

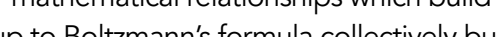
cher systems individually.

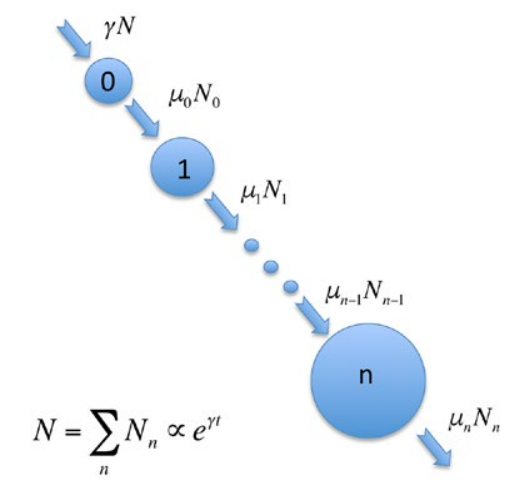

Probability Dynamics

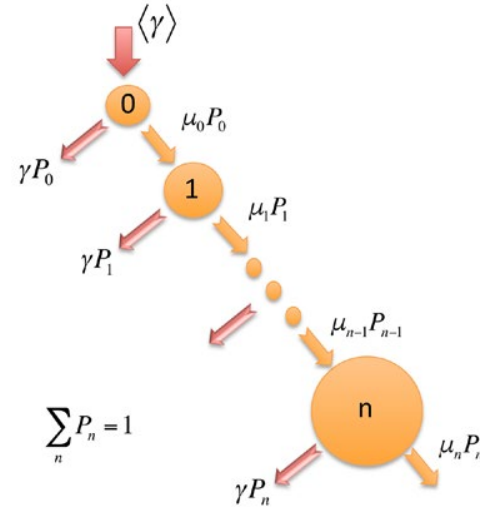

Schematic representation of the coarse-grained random growth model. Previously published in Zoltán
Néda et al hl. htpps:/doi.org /10.1371/jijurnal.pone.0179656 and is under the Creative Commons Licence 
Rescaled distribution of the citation (share) numbers.

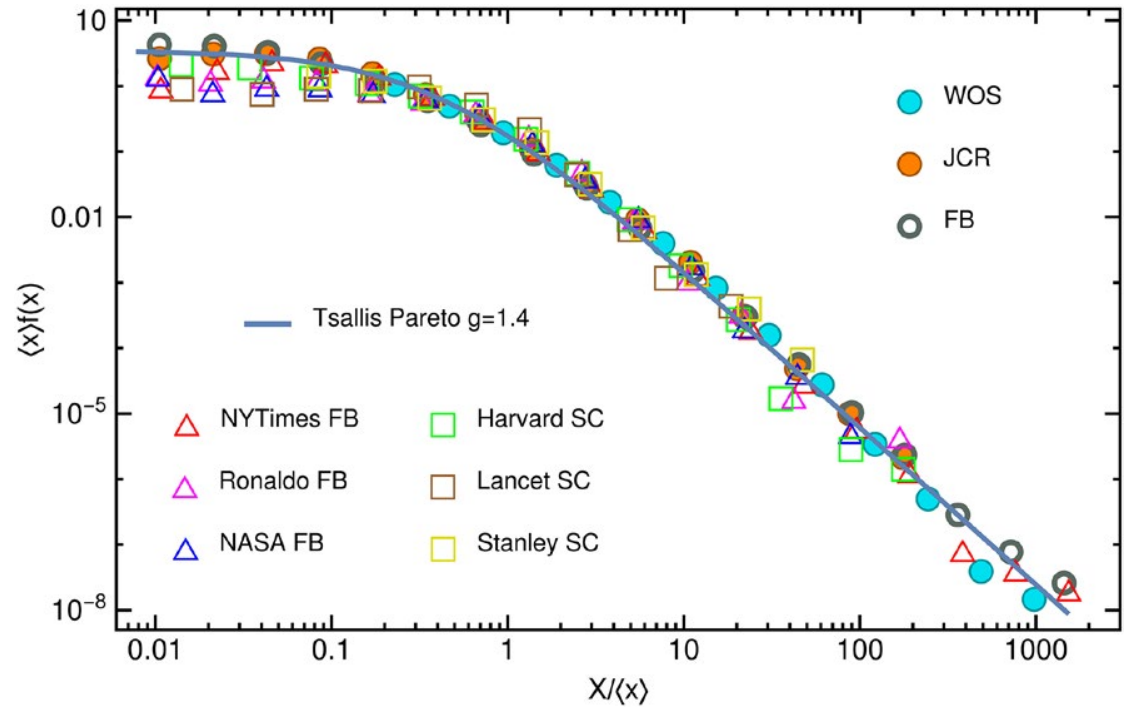

Previously published in Zoltán Néda et al. https://doi.org/10.1371/journal.pone.0179656 and is under
the Creative Commons Licence CC BY 40

This allowed the researchers to approach the hadronization problem from a new angle; providing new insights into how in the earliest moments of the universe. Dr Biró and colleagues also applied their mathematics to other situations; testing the effectiveness of their model on systems including patterns of popularity on social media, distributions in income and wealth, along with size distribution

Through these studies, Dr Biró and

A

generalizing Boltzmann's original

formula using mathematics adapted

from the principles of statistical physics

which Tsallis first generalized for the

case of entropy. As Dr Biró explains,

"We found that nonlinear master

equations: dynamical equations treating

the probabilities of systems being

in given states not linearly, but via some

use of non-logarithmic formulas for

the entropy." Using such sophisticated

mathematics, the researchers could

construct entropic probability distributions which more closely reflect those which can be observed in nature.

Ultimately, these master equations allowed Dr Biró and colleagues to

model how the probability distributions of systems evolve as time progresses, and entropy increases. Over time, an increase in entropy will mean the probability distribution associated with a system can be expected to shift. "O way of showing this relied on the use of an 'entropic divergence': a nontwo probablity distribula between continues. "Stable nonlinear master equation models describe a change of probabilities always towards a no-morechanging, stationary distribution." As such, the team's master equations could model the changes in entropy of real

systems over time.

Through this work, Dr Biró and colleagues could construct a 'nonextensive' entropy formula, which accounted for how the probability

Dr Biró and colleagues have made strides towards generalizing Boltzmann's original formula.

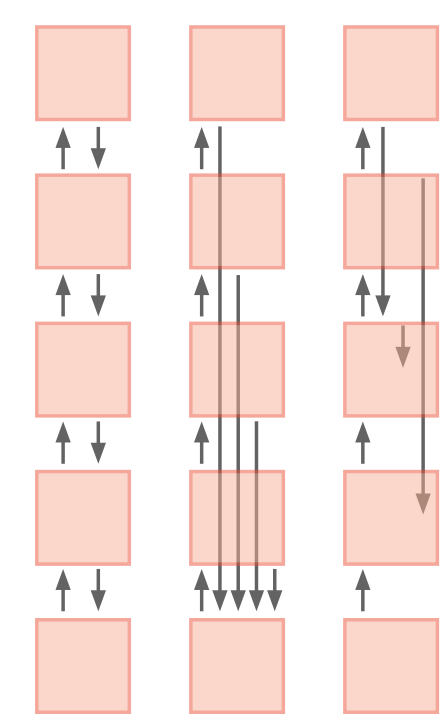

left: scheme for processes with local changes processes with resests to the ground state le.g. popularity). Right: scheme for general processes

has played out will be independent of the initial probability distribution for most materials. Since this entropic distance relationship more closely reflected reality, the master equation could be used to construct a highly generalized formula for calculating entropy. "A number of physical systems are described by stochastic master equations," explains Dr Biró "Their corresponding stationary distributions belong to maximal entropy therefore, entropy can also be defined the uniform distribution."

\section{APPLYING THE MODEL}

The mathematics described by Dr Biró and colleagues can already be used to in a wide variety of different systems. "We apply our mathematical model to various physical systems, including complex networks of popularity like citations or Facebook likes, city size distributions, produced hadron energies in big collider experiments, student exchange networks between universities, among others," Dr Biró concludes. Yet the team's mathematica description is still far from complete. In future work, the researchers will study more complex nonlinear master equations; enabling for an even greater

\section{Dr Tamás Biró}

E: Biro.Tamas@wigner.mta.hu T: +36204351283 W: www.rmki.kfki.hu/ $\sim$ sbiro

\section{Research Objectives}

Biró and his colleagues are using statistics to study problems as diverse as the formation of hadrons, changes in biodiversity, and patterns in popularity on Facebook.

\section{Detail}

H-2081 Piliscsaba, Tó sétány 5A, Hungary

Tamás studied physics at Eotvos University from 1975 til 1980, and received an MSc in physics and biophysics. He later received his $\mathrm{PhD}$ degree at the same university. Tamás is the vice director of the Institute for Particle and Nuclear Physics, the half of the Wigner Research Centre for Physics, in Budapest.

\section{Funding}

for Innovation and Technology Office (both in Hungary)

\section{Collaborators}

uj, Romania)

- András Telcs (Wigner RCP, Budapest, Hungary)

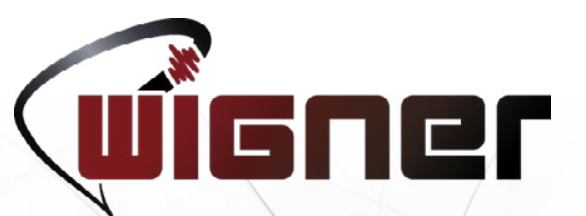

Îㄷำ

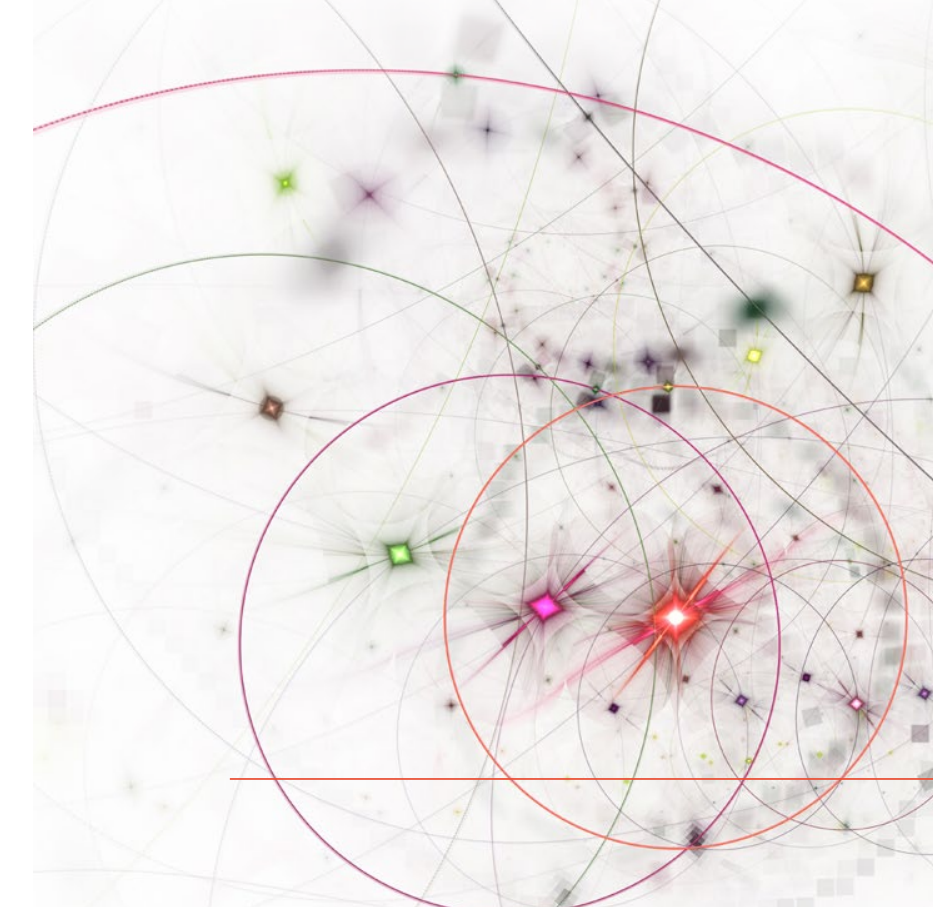

\section{References}

Biró, T.S., Barnaföldi, G.G., Biró, G., Shen, K.M. (2017). 'Near and Far from Equilibrium Power-Law Statistics'. Journal of Physics: Conference Series, 779(1), 012081.

Biró, T.S., Schram, Z., Jenkovszky, L. (2018). 'Entropy production during hadronization of a quark-gluon
plasma'. The European Physical Journal A, 54(2), 17 Biró, T.S., Neda, Z. (2018). 'Unidirectional random growth with resetting'. Physica A: Statistical Mechanic growth with resetting'.

Biró, T.S., Telcs, A., Néda, Z. (2018). 'Entropic Distance for Nonlinear Master Equation'. Universe, 4(1), 10.

\section{Personal Response}

\section{What future research studies do you have planned?}

II So far, we have discovered that whenever a

dynamical master equation uses non-linear functions
of probabilities, then the general way of constructing entropic divergence and through this the entropy probability relation leads to new formulae. On the other hand, more trivially, transition rates depending rates) also determine the stationary distribution having a non-exponential, non-Boltzmannian form.

Our plans include to study systems where both transition rate between them. Here the increase of entropy is highly a nontrivial question, and no definite answer has been found so far - except for cases restricted by the detailed balance condition. Another future development is to study processes where no

a jumps o physical systems can show avalanches or earthquakes. For these questions, we concentrate not on the final distribution of stress (which describes a state), in the dye distribution of the size of the jumps 